\title{
RELATOS DE PESQUISAS
PERSPECTIVAS DE ESTUDOS SOBRE GESTÃO DO
CONHECIMENTO NO SETOR DA MODA E DO
VESTUÁRIO, POR MEIO DE ANÁLISE SISTEMÁTICA DE
LITERATURA
}

\author{
Marcio José Silva \\ Mestre em Gestão do Conhecimento nas Organizações pelo Centro \\ Universitário de Maringá, Brasil. Professor da Universidade Estadual de \\ Maringá, Brasil. \\ E-mail: $\underline{\text { mjsilva2@uem.br }}$ \\ Cláudia Herrero Martins Menegassi \\ Doutora em Administração pela Universidade Positivo, Brasil. Professora \\ do Centro Universitário de Maringá, Brasil. \\ E-mail: claudiaherrero@gmail.com
}

\begin{abstract}
Resumo
O setor do vestuário é expressivo no ponto de vista econômico do Brasil, que é o quarto maior produtor do mundo. A criação, compartilhamento e aplicação de técnicas de gestão do conhecimento (GC) contribuem, tanto para a competitividade, quanto para o aprendizado organizacional e vêm sendo utilizados como estratégia competitiva e de inovação nas empresas. O objetivo desta pesquisa é realizar uma revisão bibliográfica sistemática de literatura, para identificar os estudos publicados em periódicos nacionais e internacionais, bem como teses e dissertações que tratam sobre a GC ou do conhecimento nas indústrias ligadas ao setor de moda e vestuário. A análise das publicações encontradas dividiu-se em: relação das publicações encontradas; palavras-chave utilizadas; objetivos e metodologia utilizados nas publicações encontradas. Os resultados mostram os estudos encontrados entre os anos de 2003 e 2015, a existência de variadas formas de abordagem quanto aos objetivos de pesquisa, bem como aponta as estratégias e tipos de pesquisa mais utilizados e lacunas para pesquisas futuras.
\end{abstract}

Palavras-chave: Conhecimento. Gestão do Conhecimento. Indústria da Moda. Indústria do Vestuário.

\section{PERSPECTIVES OF STUDIES ON KNOWLEDGE MANAGEMENT IN THE FASHION AND CLOTHING SECTOR, THROUGH A SYSTEMATIC ANALYSIS OF LITERATURE}

\begin{abstract}
The clothing sector is expressive in the economic point of view of Brazil, being the fourth largest producer in the world. The creation, sharing and application of knowledge management (KM) techniques contributes, both to competitiveness and organizational learning, and has been used as a competitive and innovative strategy in companies. The objective of this research is to realize a systematic bibliographical review of literature to identify the studies published in national and international journals, as well as theses and dissertations dealing with the KM or knowledge in the industries related to the fashion and clothing sector. The analysis of the publications found was divided in: relation of the researches found; keywords used; objectives and methodology used in the publications found. The results show the studies found between the years 2003 and 2015, the existence of various ways of approach regarding the research objectives, as well as points out the strategies and types of research most used and gaps to future researches.
\end{abstract}

Keywords: Knowledge. Knowledge Management. Fashion Industry. Clothing Industry.

Perspectivas em Gestão \& Conhecimento, João Pessoa, v. 8, n. 3, p. 192-208, set./dez. 2018. DOI: http://dx.doi.org/10.21714/2236-417X2018v8n3p192

http://periodicos.ufpb.br/ojs2/index.php/pgc. ISSN: 2236-417X. Publicação sob Licença (cc) EY-NC-ND 


\section{INTRODUÇÃO}

O setor de moda e vestuário possui grande importância pelo ponto de vista econômico do Brasil, principalmente por sua grande contribuição na geração de emprego e renda, já que é o segundo maior empregador da indústria de transformação no país (ABIT, 2016). Apesar disso essas organizações são compostas por fragmentação de processos produtivos, mix de produtos extenso, curto ciclo de vida dos produtos, influência direta da moda e imprevisibilidade de demanda atrelado a um segmento altamente competitivo movido por tendências cíclicas com influência direta da moda (FINARDI; SILVA; VARVAKIS, 2016; SILVA; MENEGASSI; PEGINO, 2017).

Segundo Jones (2005) a competitividade do setor se dá pela oferta de produtos que contenham novidades, mesmo que sutis, e isso se dá a partir das tendências de moda. A moda neste sentido, é o que direciona a criação e produção dos produtos em ciclos cada vez menores, sendo o tempo um dos principais fatores negativos para esse tipo de negócio. Nesse contexto a autora salienta que a indústria do vestuário necessita de cronogramas alinhados a todas as fases de seu ciclo produtivo, que deve contemplar atividades desde a criação até a entrega final dos produtos.

Marchi e Nardin (2009) sugerem que a transferência de conhecimento auxilia empresas criativas (ligadas à moda e ao vestuário) na organização dos processos que estão relacionados a oferta de novos produtos. Neste caso, o conhecimento contribui para ações acertáveis em relação ao curto ciclo produtivo. No entanto, para a eficiência de resultados, este conhecimento deve ser corretamente promovido, capturado, gerenciado e direcionado aos objetivos organizacionais, vindo a tornar-se então uma estratégia competitiva. Silva, Menegassi e Pegino (2017), apontam que dentre variados fatores, como a implantação de sistemas de informação e capacitação da equipe, o conhecimento é um dos principais ativos disponíveis para que essas empresas se mantenham competitivas no mercado.

Para que esse conhecimento seja utilizado é necessário a utilização de um processo sistematizado com ações que proporcionem seu melhor uso nas organizações. Esse processo sistematizado é definido por teóricos como GC. A GC possibilita que os processos de criação, compartilhamento, armazenamento e aplicação do conhecimento venham a se tornar úteis e aplicáveis a um ambiente organizacional, promovendo o aprendizado organizacional e tornando pessoas e organizações mais produtivas (DALKIR, 2011).

Segundo Vasconcelos et al. (2012) a capacidade de identificar e gerir o conhecimento vem a ser uma das mais importantes ferramentas de vantagem competitiva para as organizações contemporâneas. Neste sentido uma organização é vista como um espaço propício para os processos de criação, compartilhamento, armazenamento e aplicação do conhecimento. Lastres e Ferraz (1999) indicam que o conhecimento vem sendo utilizado por organizações como a principal fonte de maximização de resultados.

No ambiente organizacional sugere-se que o conhecimento desponte inicialmente de modo tácito, ou o conhecimento individual, que após identificado pode se tornar explicito, sistematizado. Ao explicitar o conhecimento e, assim, compartilhá-lo nas organizações, este pode proporcionar vantagens ou ganhos mesmo que inicialmente intangíveis para a gestão dos processos, principalmente se vinculados ao aprendizado organizacional (DALKIR, 2011; VASCONCELOS et al. 2012).

Nesse sentido, os processos e ferramentas que compõem uma sistemática de GC, se aplicam a diferentes tipos de organizações. Desta forma, o setor de moda e vestuário, tem a possibilidade de se utilizar desse sistema de gestão, agregando valor a seus processos produtivos. Ao considerar que este setor tem como uma de suas características a inovação, necessita de meios que auxiliem em propor inovação e a troca rápida de informações (MARCHI; NARDIN, 2009; 2014).

Perspectivas em Gestão \& Conhecimento, João Pessoa, v. 8, n. 3, p. 192-208, set./dez. 2018. 
Diante do contexto apresentado, o objetivo desta pesquisa é realizar uma análise sistemática de literatura, com foco na seguinte questão: quais estudos publicados em periódicos nacionais e internacionais, bem como teses e dissertações, tratam sobre 0 conhecimento em indústrias do setor de moda e vestuário?

\section{SETOR DE MODA E VESTUÁRIO E A GESTÃO DO CONHECIMENTO}

De acordo com a Associação Brasileira das Indústrias Têxteis e de Vestuário (ABIT, 2016), a indústria do vestuário apresenta números que a tornam expressiva para a economia brasileira. Os dados apresentados demonstram que o país produz aproximadamente 5,3 bilhões de peças de vestuário ao ano. Para atender tal demanda, o Brasil conta com o quarto maior parque produtivo de vestuário do mundo tendo cerca de 30 mil empresas. Em levantamento apresentado pela ABIT (2016), essas indústrias faturaram US\$ 36,2 bilhões no ano de 2015, e que o setor é segundo maior empregador da indústria de transformação.

De acordo com a ABIT (2016), a cadeia têxtil e de confecção se convergem, pois, fazem parte da mesma cadeia produtiva. No entanto, diferem no sentido de a primeira corresponder às empresas produtoras de fibras naturais, artificiais e sintéticas que serão beneficiadas por fiações e tecelagens gerando materiais têxteis; já a segunda, está relacionada à indústria de transformação dos artigos têxteis em produtos do vestuário, cama mesa e banho. Neste trabalho o objeto de estudo são as indústrias que produzem vestuário.

Baxter (1998) aponta que os produtos do vestuário são concebidos mediante inovação, seguindo interferências rápidas ocasionadas pelo sistema da moda, que é apresentado nos produtos por meio de tendências cíclicas ligadas às estações climáticas, primavera, verão, outono e inverno. A moda, de acordo com Jones (2005), dá um sentido de ordem à concepção de produtos, e as indústrias ligadas ao vestuário, consequentemente, são guiadas por essas tendências. Silva, Menegassi e Pegino (2017) identificam que o setor é altamente influenciado por fatores que interferem diretamente na tomada de decisões, gestão do tempo e da informação.

Nesse sentido, observa-se que é um segmento composto por um grande número de processos, pessoas e informações em trânsito (FINARDI; SILVA; VARVAKIS, 2016). Nesse sentido Silva, Menegassi e Pegino (2017) tratam que nessas empresas os setores estão interligados e as informações e conhecimento ali existentes podem atuar no sentido de estratégia competitiva. Marchi e Nardin (2009) sugerem que a transferência de conhecimento auxilia empresas criativas (ligadas à moda e ao vestuário) na concepção de novos produtos e organização de seus processos produtivos. Neste caso, o conhecimento pode contribuir para ações que auxiliem essas empresas a atingirem seus objetivos organizacionais.

Para Lastres e Ferraz (2010) o conhecimento pode ser considerado como algo intangível ou não material, não sendo esse ativo esgotável ou deteriorável, seu consumo não o destrói; se descartado não deixa resíduos físicos e, caso cedido ou transferido, não será perdido. Com essa percepção Wiig (1997) aponta que fatores naturais contribuíram para que o conhecimento existente nas organizações passasse a ser gerido. Após esforços teóricos sobre o assunto, o processo foi direcionado a uma sistematização conhecido como GC. De modo geral, esse processo tem por objetivo aprimorar e gerar maior competitividade, por meio dos ativos de conhecimento.

As organizações esperam na GC a geração de resultados, no entanto é preciso que haja tentativas para gerir o conhecimento e nem sempre deve se esperar resultados a curto prazo. Para isto, é necessário que se implante uma fase de experimento, incubação e um estudo direcionado que vincule os objetivos organizacionais com o que a GC pode proporcionar (CEN, 2004). A GC no contexto da moda e do vestuário pode apresentar resultados importantes no sentido de gestão dos processos produtivos, considerando a grande interação entre setores e

Perspectivas em Gestão \& Conhecimento, João Pessoa, v. 8, n. 3, p. 192-208, set./dez. 2018. 
que por se tratar de um setor que tem forte relação com a criatividade, as atividades ligadas a interação entre os indivíduos mostram-se como favoráveis para o uso compartilhado do conhecimento existente nesse tipo de organização (LI et al. 2005; OLARU et al. 2013).

Para Dalkir (2011), a capacidade de gerir o conhecimento é algo decisivo em uma economia que prioriza o conhecimento como fator primordial de competitividade. De acordo com a autora, para que isso ocorra, é necessário que a organização identifique, gere, adquira, difunda e capture os benefícios do conhecimento, a partir disso, pode torna-lo então um fator de estratégia. Deste modo a GC apresenta atividades como modelos, diagnósticos, práticas, ciclos, ferramentas, que norteiam a melhor forma de tratar o conhecimento dentro de uma organização.

\section{PROCEDIMENTO METODOLÓGICO}

A pesquisa bibliográfica de estratégia sistemática baseia-se em um estudo que compreende toda a bibliografia publicada disponível sobre determinado assunto, a partir da escolha de um tema que deve gerar um problema a ser resolvido. Por meio do levantamento preliminar da área de estudo se formula um problema de pesquisa coerente que possa ser respondido (MARCONI; LAKATOS, 2006, GIL, 2010). De acordo com Gil (2010), este tipo de estudo possibilita uma melhor visão sobre o problema de pesquisa e quais podem ser os direcionamentos futuros sobre o mesmo.

Deste modo foi realizada uma revisão sistemática de materiais bibliográficos já publicados sobre o assunto, seguindo o seguinte critério: 1 . Formulação da questão para o problema; 2 . Escolha dos termos a serem pesquisados; 3 . Seleção das bases de busca científica para levantamento de publicações; 4. Seleção dos materiais a serem utilizados; 5 . Análise e apresentação dos resultados; e, 6 . Considerações finais.

Uma vez determinado o procedimento metodológico, as bases de busca científica utilizadas para o levantamento dos dados são: Periódicos Capes; Scielo; Emerald Insight; BDTD (Biblioteca Digital Brasileira de Teses e Dissertações); Science Direct, Scopus e, por último, o Scholar. As bases Periódicos Capes, Scielo, Emerald Insight, Science Direct e Scopus foram determinadas por conter um grande número de publicações que apresentam importância e relevância científica para a área de GC, deste modo a busca nessas bases contribuiu para se obter a maior parte dos trabalhos publicados sobre o assunto. A BDTD foi utilizada por conter especificamente o acervo sobre teses e dissertações, o que contribui para visualizar o panorama das pesquisas científicas sobre o tema. E por último o Scholar foi utilizado para a obtenção de publicações não disponíveis de modo público nas bases anteriormente mencionadas.

Para a definição dos termos de busca levou-se em consideração a GC e a indústria de moda/vestuário, levando em conta que de acordo com a ABIT (2016) essa indústria está inserida na cadeia têxtil, o termo têxtil também foi utilizado para a pesquisa. As palavras-chave que compuseram a busca foram: gestão do conhecimento + indústria do vestuário; gestão do conhecimento + indústria + vestuário; gestão do conhecimento + moda; gestão do conhecimento + indústria + moda; gestão do conhecimento + indústria + têxtil + vestuário. Foi feita a pesquisa com as variantes dessas palavras em Inglês, sendo: knowledge management + clothing industr*1; knowledge management + fashion industr*, não se utilizou a variante para têxtil. Os resultados encontrados foram:

\footnotetext{
${ }^{1}$ Caractere booleano que suprime uma letra ou termo, assim não é necessário usar variações no plural.
} 
Quadro 1 - Relação das publicações encontradas por base de busca referente a primeira etapa de pesquisa

\begin{tabular}{|l|c|c|}
\hline \multicolumn{1}{|c|}{ Base de Busca } & $\begin{array}{c}\text { Quantidade de trabalhos } \\
\text { encontrados }\end{array}$ & Quantidade de trabalhos utilizados \\
\hline Periódicos Capes & 16 & 5 \\
\hline Scielo & 2 & 0 \\
\hline Emerald Insight & 0 & 0 \\
\hline BDTD & 24 & 2 \\
\hline Science Direct & 0 & 0 \\
\hline Scopus & 0 & 0 \\
\hline Scholar & 0 & 1 \\
\hline Total & $\mathbf{4 2}$ & $\mathbf{8}$ \\
\hline
\end{tabular}

Fonte: Elaborado pelos autores (2017)

Essa primeira busca não gerou uma quantidade satisfatória de resultados, então o processo foi refeito, utilizando apenas a palavra conhecimento em sua variante em Inglês, sendo: knowledge + clothing industr*; knowledge + fashion industr*; "knowledge" + "clothing" + industr*; "knowledge" + "fashion" + industr*. Assim, houve um acréscimo nos resultados encontrados, já que estudos que abordam apenas uma forma de aplicação ou utilização do conhecimento, ou ainda, apenas alguma etapa específica de uma sistemática de GC, não foram contemplados na busca inicial.

Quadro 2 - Relação das publicações encontradas por base de busca referente aos termos em Inglês

\begin{tabular}{|l|c|c|}
\hline \multicolumn{1}{|c|}{ Base de Busca } & $\begin{array}{c}\text { Quantidade de trabalhos } \\
\text { encontrados }\end{array}$ & Quantidade de trabalhos utilizados \\
\hline Periódicos Capes & 21 & 5 \\
\hline Scielo & 1 & 0 \\
\hline Emerald Insight & 58 & 5 \\
\hline BDTD & 6 & 1 \\
\hline Science Direct & 8 & 3 \\
\hline Scopus & 3 & 0 \\
\hline Scholar & 0 & 1 \\
\hline Total & $\mathbf{9 7}$ & $\mathbf{1 5}$ \\
\hline
\end{tabular}

Fonte: Elaborado pelos autores (2017)

As palavras-chave utilizadas para busca deveriam estar presentes, no título ou no assunto dos trabalhos. Desse modo, as buscas geraram resultados de publicações entre os anos de 2003 e 2015, com um total de 23 trabalhos, em forma de artigos científicos (20 resultados) e teses (03 resultados). Para a seleção dos trabalhos utilizados realizou-se a leitura do título e do resumo dos mesmos, onde foi observado que apesar da utilização de caracteres de seleção haviam trabalhos que não se encaixavam no escopo desta pesquisa por apresentarem temáticas gerais sobre a GC e não específicas sobre o setor de moda/vestuário. A pesquisa foi realizada no período entre abril e junho de 2016.

\section{APRESENTAÇÃO DOS RESULTADOS E DISCUSSÃO}

Diante do exposto até aqui, foi realizada a busca que priorizou identificar como a literatura trata sobre a GC e sua relação com indústrias de moda e vestuário. É importante destacar que a moda possui relação muito próxima com o setor de vestuário, deste modo os trabalhos que tratam sobre a moda, foram considerados como relevantes para esta análise. Outro fator que deve ser observado, é que como a GC é composta por atividades que

Perspectivas em Gestão \& Conhecimento, João Pessoa, v. 8, n. 3, p. 192-208, set./dez. 2018. 
promovem seus processos de criação, compartilhamento, armazenamento e aplicação do conhecimento, há a existência de publicações que discorrem apenas sobre um desses processos ou alguma outra atividade ligada a uma sistemática de GC.

É possível observar diante dos resultados encontrados, o ano do primeiro trabalho publicado e disponível para consulta, a primeira publicação em português, quais os tipos dos trabalhos, quais seus objetivos e ainda algumas lacunas que podem ser atendidas com futuras pesquisas.

Quadro 3 - Relação das publicações encontradas por tipo, autor/ano e título

\begin{tabular}{|c|c|c|}
\hline Tipo & Autor/ano & Título \\
\hline Tese & Weller (2003) & $\begin{array}{l}\text { Fashion's influence on garment mass production: knowledge, } \\
\text { commodities and the capture of value (Influência da moda na } \\
\text { produção do vestuário em massa: conhecimento, comodites e } \\
\text { captura de valor). }\end{array}$ \\
\hline Artigo & $\begin{array}{l}\text { Bertolini et al. } \\
\text { (2004) }\end{array}$ & $\begin{array}{l}\text { Requeriments of na ERP enterprise modeller for optimally } \\
\text { managing the fashion industry suplly chain (Requisitos de um } \\
\text { modelo ERP para otimizar a gestão da cadeia de suprimentos da } \\
\text { indústria de moda). }\end{array}$ \\
\hline Artigo & Li et al. (2005) & $\begin{array}{l}\text { Managing Knowledge in Fashion Businesses: A Contextual Review } \\
\text { (A gestão do conhecimento em empresas de moda: Uma análise } \\
\text { contextual). }\end{array}$ \\
\hline Artigo & Li et al. (2006) & $\begin{array}{l}\text { Managing Knowledge in Fashion Businesses: A Survey of } \\
\text { Contemporary Tools for Coordination and Collaboration (A gestão } \\
\text { do conhecimento em empresas de Moda: Uma pesquisa de } \\
\text { ferramentas contemporâneas para coordenação e colaboração). }\end{array}$ \\
\hline Artigo & Weller (2007) & $\begin{array}{l}\text { Fashion as viscous knowledge: fashion's role in shaping trans- } \\
\text { national garment production (Moda como conhecimento viscoso: a } \\
\text { função da moda na formação da produção de vestuário } \\
\text { transnacional). }\end{array}$ \\
\hline Artigo & $\begin{array}{l}\text { Balestrin, Vargas } \\
\text { e Fayard, (2008) }\end{array}$ & $\begin{array}{l}\text { Knowledge creation in small-firm network (Criação do } \\
\text { Conhecimento em rede de pequenas empresas). }\end{array}$ \\
\hline Artigo & $\begin{array}{l}\text { Davey, } \\
\text { Schneider e } \\
\text { Davey (2009) }\end{array}$ & $\begin{array}{l}\text { Intellectual capital disclousure and the fashion industry (Divulgação } \\
\text { do capital intelectual na indústria de moda). }\end{array}$ \\
\hline Artigo & $\begin{array}{l}\text { Marchi e Nardin } \\
\text { (2009) }\end{array}$ & $\begin{array}{l}\text { Market Knowledge Transfer and Time Pressure in new Product } \\
\text { Development: The Emergent Role of Knowledge Intermediaries in } \\
\text { Fashion Industry (Mercado de transferência de conhecimento e } \\
\text { pressão do tempo no desenvolvimento de novos produtos: o papel } \\
\text { emergente do conhecimento intermediário na indústria da moda). }\end{array}$ \\
\hline Artigo & Fraganito (2010) & $\begin{array}{l}\text { Cooperação e Inovação no Processo de Criação e Gestão do } \\
\text { Conhecimento no sector Têxtil e de Vestuário. }\end{array}$ \\
\hline Tese & Tres (2010) & $\begin{array}{l}\text { A dinâmica dos fluxos de conhecimentos locais e externos no } \\
\text { sistema local de produção têxtil-vestuário catarinense. }\end{array}$ \\
\hline Artigo & $\begin{array}{l}\text { Li, Xiaogang e } \\
\text { Jun (2010) }\end{array}$ & $\begin{array}{l}\text { Apparel Brand Image Management Based on Knowledge } \\
\text { Management (Gerenciamento da marca de vestuário baseada na } \\
\text { Gestão do Conhecimento). }\end{array}$ \\
\hline Artigo & $\begin{array}{l}\text { Cavalcante et al. } \\
(2011)\end{array}$ & $\begin{array}{l}\text { A abordagem prospectiva de gestão do conhecimento na indústria } \\
\text { da moda: um modelo para o aumento de inovação de produto. }\end{array}$ \\
\hline Tese & $\begin{array}{l}\text { Beirão Filho } \\
(2011)\end{array}$ & $\begin{array}{l}\text { Criação e compartilhamento do conhecimento na área de moda em } \\
\text { um sistema virtual integrado de informações. }\end{array}$ \\
\hline Artigo & Olszowy (2012) & $\begin{array}{l}\text { Importance of Intellectual Capital in Enterprise Growth, with Special } \\
\text { Emphasis on the Textile and Clothing Industry in Poland }\end{array}$ \\
\hline
\end{tabular}

Perspectivas em Gestão \& Conhecimento, João Pessoa, v. 8, n. 3, p. 192-208, set./dez. 2018. 


\begin{tabular}{|c|c|c|}
\hline & & $\begin{array}{l}\text { (Importância do capital intelectual no crescimento das empresas, } \\
\text { com especial ênfase para a indústria têxtil e de vestuário na } \\
\text { Polónia). }\end{array}$ \\
\hline Artigo & $\begin{array}{l}\text { Olaru, } \\
\text { Ghituleasa, } \\
\text { Filipescu (2012) }\end{array}$ & $\begin{array}{l}\text { Knowledge Management in the Fashion Industry (Gestão do } \\
\text { Conhecimento na Indústria de Moda) }\end{array}$ \\
\hline Artigo & Pires (2012) & $\begin{array}{l}\text { Cultura organizacional e memória empresarial na indústria da } \\
\text { moda: gestão de ativos do conhecimento como estratégia } \\
\text { corporativa para a construção de identidades de marcas. }\end{array}$ \\
\hline Artigo & $\begin{array}{l}\text { Olaru et al. } \\
\text { (2013) }\end{array}$ & $\begin{array}{l}\text { Collaborative Technologies and knowledge management in the } \\
\text { fashion industry (Tecnologias colaborativas e gestão do } \\
\text { conhecimento na indústria de moda). }\end{array}$ \\
\hline Artigo & $\begin{array}{l}\text { Sohn, Vieira e } \\
\text { Filho, (2013) }\end{array}$ & $\begin{array}{l}\text { Estudo comparativo de canais de transmissão de conhecimento em } \\
\text { clusters têxteis e de vestuário de Santa Catarina - Brasil e Norte de } \\
\text { Portugal. }\end{array}$ \\
\hline Artigo & $\begin{array}{l}\text { Paulos, Moniz } \\
(2013)\end{array}$ & $\begin{array}{l}\text { Os trabalhadores do conhecimento num setor tradicional - O caso } \\
\text { dos designers do vestuário. }\end{array}$ \\
\hline Artigo & $\begin{array}{l}\text { Marchi, Nardin } \\
(2014)\end{array}$ & $\begin{array}{l}\text { Organizing Creativity in the Italian Fashion Industry (Organizando a } \\
\text { criatividade na indústria de moda italiana). }\end{array}$ \\
\hline Artigo & $\begin{array}{l}\text { Oliveira, } \\
\text { Epaminondas } \\
(2014)\end{array}$ & $\begin{array}{l}\text { Conhecimento, inovação e estratégia competitiva: um estudo do } \\
\text { setor atacadista de moda. }\end{array}$ \\
\hline Artigo & $\begin{array}{l}\text { Kanat, Altigan } \\
\text { (2014) }\end{array}$ & $\begin{array}{l}\text { Effects of Knowledge Management on Supply Chain Management } \\
\text { in the Clothing Sector: Turkish Case (Efeitos da Gestão do } \\
\text { Conhecimento na Gestão da cadeia de suprimentos no setor do } \\
\text { vestuário: Caso Turco). }\end{array}$ \\
\hline Artigo & $\begin{array}{l}\text { Marcella, } \\
\text { Rowley (2015) }\end{array}$ & $\begin{array}{l}\text { An exploration of the extent to which project management tools } \\
\text { and techniques can be applied across creative industries through a } \\
\text { study of their application in the fashion industry in the North East of } \\
\text { Scotland (Uma exploração da extensão em que ferramentas e } \\
\text { técnicas de gerenciamento de projetos podem ser aplicadas em } \\
\text { indústrias criativas, através de um estudo de sua aplicação na } \\
\text { indústria da moda no Nordeste da Escócia). }\end{array}$ \\
\hline
\end{tabular}

Fonte: Elaborado pelos autores (2017)

O Quadro 3 apresenta os trabalhos encontrados e que de algum modo abordam sobre o conhecimento ou a GC e indústrias de moda e vestuário. Nota-se que as publicações são apresentadas de maneira não uniforme, entre os anos de 2003 e 2015. Percebe-se ainda uma certa escassez de publicações sobre o tema, o que sugere um espaço para atender a gaps teóricos em relação a esse assunto. Ao analisar os títulos, percebe-se que os trabalhos propõem diferentes abordagens de pesquisa como, relação com o capital intelectual, melhoria de processos, inovação e estratégia.

Observa-se ainda no Quadro 3 o idioma de publicação das pesquisas. Dos trabalhos selecionados 15 estão escritos em Inglês, sete em Português e o trabalho de Fraganito (2010) está escrito em Espanhol, no entanto está publicado em um repositório português, assim o título do mesmo também se apresenta nesse idioma. O primeiro trabalho escrito em Português foi a tese de Tres (2010). Nota-se certo retardo na abordagem do assunto em publicações brasileiras em relação às publicações em inglês, que, são datadas em seis anos antes. Destaca-se que Paulos e Moniz (2013) são pesquisadores do Reino Unido e de Portugal, respectivamente, e publicaram seu trabalho em uma revista no Brasil com o idioma em português.

Perspectivas em Gestão \& Conhecimento, João Pessoa, v. 8, n. 3, p. 192-208, set./dez. 2018. 
A pesquisa mais recente encontrada é a de Marcella e Rowley (2015), enquanto o trabalho de Weller (2003) é o resultado mais distante, e se faz presente com mais uma publicação no ano de 2007. O autor Li, aparece três vezes nos resultados, sendo, Li et al. (2005, 2006) e Li; Xiaogang e Jun (2010). Marchi e Nardin aparecem com duas publicações, sendo a primeira em 2009 e, a segunda, em 2014, sugerindo a continuidade das investigações, por parte desses autores. Não foram encontrados outros trabalhos que tratem do tema proposto dos demais autores. A pesquisa identificou um certo caráter de novidade sobre a discussão entre a GC e o setor de moda/vestuário, havendo ligeiro crescimento de publicações no ano de 2009, e nos demais anos o número de publicações se encontra na casa de duas ao ano.

Diante do encontrado, avaliando o teor do conteúdo, espaço de tempo entre as publicações e poucas publicações que mostram continuidade nas pesquisas, isso mostra relação ao que trata Wiig (1997), sobre o fato de a GC se apresentar como algo novo no sentido de estudos científicos, já que inicialmente foi tratada como uma ferramenta de gestão do conhecimento organizacional com o objetivo de promover resultados com base nos ativos de conhecimento.

O uso de palavras-chave contribui para a indexação das pesquisas e apresentar os conceitos principais do assunto, e nesse sentido verificou-se quais palavras-chave foram indicadas por seus autores nas publicações. Com isso é possível verificar se os autores atribuem termos específicos de elementos de GC ou remetem à própria GC. As palavras-chave indicadas nas publicações são apresentadas no Quadro 4.

Quadro 4 - Palavras-chave indicadas nas publicações

\begin{tabular}{|c|c|}
\hline Autor/Ano & Palavras-chave \\
\hline $\begin{array}{l}\text { Weller (2003), Marchi; Nardin } \\
\text { (2014) }\end{array}$ & Não apresenta. \\
\hline Bertolini et al. (2004) & $\begin{array}{l}\text { Manufacturing resource planning; Fashion industry; Production } \\
\text { planning and control. }\end{array}$ \\
\hline Li et al. (2005) & $\begin{array}{l}\text { Knowledge; Knowledge management; Coordination and } \\
\text { Collaboration. }\end{array}$ \\
\hline Li et al. (2006) & $\begin{array}{l}\text { Knowledge; Knowledge management; Communication; } \\
\text { Collaboration; Coordination Technologies. }\end{array}$ \\
\hline Weller (2007) & Knowledge; Fashion industries; Economic geography. \\
\hline $\begin{array}{l}\text { Balestrin; Vargas e Fayard, } \\
\text { (2008) }\end{array}$ & Knowledge creation; Small enterprises; Knowledge management. \\
\hline Marchi; Nardin (2009) & $\begin{array}{l}\text { Knowledge transfer; New product development; Cognitives } \\
\text { distance; Time; Knowledge intermediaries. }\end{array}$ \\
\hline $\begin{array}{l}\text { Davey; Schneider e Davey } \\
\text { (2009) }\end{array}$ & $\begin{array}{l}\text { Intellectual capital; Brands; Fashion Industry; Disclosure; Europe; } \\
\text { North America. }\end{array}$ \\
\hline Tres (2010) & $\begin{array}{l}\text { Sistemas de manufaturas; Clusters; Conhecimento; Aprendizagem; } \\
\text { Indústria Têxtil; Vestuário. }\end{array}$ \\
\hline Fraganito (2010) & Gestão Conhecimento; Capacidade e Absorção. \\
\hline Li; Xiaogang e Jun (2010) & Apparel Brand; Knowledge Management; Brand Image Strategy. \\
\hline Cavalcante et al. (2011) & $\begin{array}{l}\text { Abordagem prospectiva; Tendências de consumo; Gestão do } \\
\text { conhecimento; Inovação de produto. }\end{array}$ \\
\hline Beirão Filho (2011) & $\begin{array}{l}\text { Gestão do Conhecimento; Tecnologia da informação e } \\
\text { comunicação; Hipermídia; Ambiente Interativo; Moda. }\end{array}$ \\
\hline $\begin{array}{l}\text { Olaru; Ghituleasa e Filipescu } \\
(2012)\end{array}$ & Knowledge management, Fashion, Customization, CAD/CAM \\
\hline Olszowy (2012) & Intellectual capital; Textille and clothing industry. \\
\hline Pires (2012) & $\begin{array}{l}\text { Memória empresarial; Gestão da informação e do Conhecimento; } \\
\text { Indústria da moda. }\end{array}$ \\
\hline
\end{tabular}

Perspectivas em Gestão \& Conhecimento, João Pessoa, v. 8, n. 3, p. 192-208, set./dez. 2018. 


\begin{tabular}{|l|l|}
\hline Paulos; Moniz (2013) & Reestruturação; Conhecimento; Vestuário; Designers. \\
\hline Olaru et al. (2013) & Knowledge management; Fashion; customization; CAD/CAM. \\
\hline Sohn; Vieira e Filho (2013) & $\begin{array}{l}\text { Clusters industriais; Aprendizagem interorganizacional; Canais de } \\
\text { transmissão do conhecimento. }\end{array}$ \\
\hline Oliveira, Epaminondas (2014) & $\begin{array}{l}\text { Conhecimento; Inovação de produtos; Estratégia funcional; Setor } \\
\text { atacadista de moda; Estudo multicaso. }\end{array}$ \\
\hline Kanat; Altigan (2014) & $\begin{array}{l}\text { Clothing Sector; Supply chain management; Knowledge } \\
\text { management; Analytic network process. }\end{array}$ \\
\hline Marcella; Rowley (2015) & $\begin{array}{l}\text { Project management; Tools; Body of Knowledge; Creative } \\
\text { Industries; Fashion; SMEs; Creative mindset; Analytical mindset; } \\
\text { Professionalism; Succes criteria; Project planning; Continuous } \\
\text { improvement. }\end{array}$ \\
\hline
\end{tabular}

Fonte: Elaborado pelos autores (2017)

As palavras-chave que apareceram com maior frequência são: knowledge management, knowledge e a palavra knowledge associada a alguma ferramenta de GC ou outros termos específicos da área de moda, totalizando 11 resultados. Ao associá-los com os termos em português, tem-se: gestão do conhecimento, conhecimento e conhecimento associado a algum outro termo, o que totalizou seis resultados. Sobre a área (moda e vestuário) as palavras-chave nas publicações em inglês mostram a indicação em cinco trabalhos com o termo fashion e dois com o termo clothing. Já nas publicações em português, três apresentam o termo moda e dois o termo vestuário. Com isso é possível perceber que no que se refere a esse tipo de pesquisa, não só a GC é priorizada, mas também o conhecimento como um elemento a ser estudado principalmente no sentido de compartilhamento. E que os termos identificados nas palavras-chave são suficientes para apresentarem os conceitos do assunto.

Após a verificação das palavras-chave foi verificado os objetivos de pesquisa. Esta análise é importante para verificar os direcionamentos já tratados nas pesquisas, abrindo a possibilidade de apresentar gaps teóricos que podem ser tratados em outras pesquisas. Os objetivos descritos pelos autores estão presentes no Quadro 5.

Quadro 5 - Objetivos indicados nas publicações encontradas

\begin{tabular}{|l|l|}
\hline \multicolumn{1}{|c|}{ Autor/Ano } & \multicolumn{1}{c|}{ Objetivo } \\
\hline Weller (2003) & $\begin{array}{l}\text { Explicar como as ideias sobre moda são incorporadas em objetos materiais } \\
\text { (vestuário) e como a produção no sentido de moda influencia a organização } \\
\text { industrial e molda as geografias de localização industrial, o comércio global e } \\
\text { a constituição de lugares. }\end{array}$ \\
\hline Bertolini et al. (2004) & $\begin{array}{l}\text { Estudar com um painel de especialistas, (acadêmicos, desenvolvedores de } \\
\text { software e usuários finais na área de suprimentos), para definir as } \\
\text { características principais que caracterizam um modelador para a indústria da } \\
\text { moda. }\end{array}$ \\
\hline Li et al. (2005) & $\begin{array}{l}\text { Investigar como implementar a gestão do conhecimento para a coordenação } \\
\text { e colaboração e quais são as questões importantes na gestão do } \\
\text { conhecimento em globalizantes contextos de negócios da moda, hoje. }\end{array}$ \\
\hline Li et al. (2006) & $\begin{array}{l}\text { Examinar as funções das mais recentes tecnologias de comunicação e de } \\
\text { coordenação e determinar como eles ajudam as organizações a } \\
\text { compartilhar, armazenar e transferir o seu "conhecimento" entre e dentro } \\
\text { das organizações. }\end{array}$ \\
\hline Weller (2007) & $\begin{array}{l}\text { Identificar as várias modalidades de conhecimento de moda e caracterizar } \\
\text { suas diferentes taxas e extensões de transmissão, através do espaço e do } \\
\text { tempo em termos da sua relativa complexidade. }\end{array}$ \\
\hline Balestrin; Vargas e Fayard, \\
(2008) & $\begin{array}{l}\text { Apontar para um entendimento de como a dinâmica de criação de } \\
\text { conhecimento ocorre dentro de uma rede de pequenas empresas. }\end{array}$ \\
\hline
\end{tabular}

Perspectivas em Gestão \& Conhecimento, João Pessoa, v. 8, n. 3, p. 192-208, set./dez. 2018. 


\begin{tabular}{|c|c|}
\hline $\begin{array}{l}\text { Davey; Schneider e Davey } \\
\text { (2009) }\end{array}$ & $\begin{array}{l}\text { Examinar a natureza e a extensão da divulgação do capital intelectual e de } \\
\text { marketing entre empresas de moda, especificamente para comparar o } \\
\text { capital intelectual }(\mathrm{Cl}) \text { e sua divulgação entre empresas e setores de } \\
\text { empresas europeias e norte-americanas de moda. }\end{array}$ \\
\hline Marchi; Nardin (2009) & $\begin{array}{l}\text { Identificar os processos de transferência do conhecimento envolvidos na } \\
\text { atividade de inovação do produto na indústria de moda. }\end{array}$ \\
\hline Fraganito (2010) & $\begin{array}{l}\text { Aprofundar o conhecimento e identificar sua importância para a } \\
\text { organização, identificando e avaliando agentes que compõem a sua rede, } \\
\text { para programar e implementar as suas etapas de planejamento estratégico } \\
\text { para manter uma relação dinâmica com a rede, abandonando a ideia de que } \\
\text { a organização é o limite de qualquer estratégia organizacional. }\end{array}$ \\
\hline Tres (2010) & $\begin{array}{l}\text { Descrever o estado em que se encontram os fabricantes de têxteis e de } \\
\text { vestuário quanto ao gerenciamento do capital intelectual em empresas na } \\
\text { Polônia. }\end{array}$ \\
\hline Li; Xiaogang e Jun (2010) & $\begin{array}{l}\text { Introduzir a gestão do conhecimento para a construção da imagem de marca } \\
\text { da indústria do vestuário e a aliança para aumentar a capacidade de } \\
\text { adquirir, integrar, compartilhar e criar conhecimento. }\end{array}$ \\
\hline Cavalcante et al. (2011) & $\begin{array}{l}\text { Apresentar um framework conceitual sobre a abordagem prospectiva para } \\
\text { operacionalizar os três processos básicos para inovação de produtos: } \\
\text { geração, divulgação e uso de informações de mercado. }\end{array}$ \\
\hline Beirão Filho (2011) & $\begin{array}{l}\text { Promover a criação e o compartilhamento do conhecimento na área de } \\
\text { moda, utilizando ferramentas da tecnologia da informação e comunicação e } \\
\text { desenvolver um sistema digital de informação de moda para a captura e } \\
\text { disseminação do conhecimento. }\end{array}$ \\
\hline Olszowy (2012) & $\begin{array}{l}\text { Determinar e avaliar a ocorrência e gestão do capital intelectual entre } \\
\text { entrevistados, tratando o capital humano e estrutural, proporcionando } \\
\text { assim, uma imagem confiável da utilização dos ativos intangíveis na indústria } \\
\text { do vestuário. }\end{array}$ \\
\hline $\begin{array}{l}\text { Olaru; Ghituleasa, Filipescu } \\
\text { (2012) }\end{array}$ & Examinar a gestão do conhecimento na indústria de moda. \\
\hline Pires (2012) & $\begin{array}{l}\text { Promover estudos, além da ideia de memória empresarial, como simples } \\
\text { prática de arquivamento da informação ou de gestão da comunicação } \\
\text { corporativa. }\end{array}$ \\
\hline Olaru et al. (2013) & Examinar a gestão do conhecimento na indústria da moda. \\
\hline Sohn; Vieira e Filho (2013) & $\begin{array}{l}\text { Identificar e analisar os canais de aprendizagem tecnológica em dois clusters } \\
\text { têxteis e de vestuário: um localizado em Santa Catarina, no Brasil, e outro, } \\
\text { na região Norte de Portugal. }\end{array}$ \\
\hline Paulos; Moniz (2013) & $\begin{array}{l}\text { Identificar qual o impacto da reestruturação do trabalho na "sociedade do } \\
\text { conhecimento", na profissão de designer do vestuário. }\end{array}$ \\
\hline Marchi; Nardin (2014) & $\begin{array}{l}\text { Investigar as forças que influenciam o gerenciamento da criatividade na } \\
\text { indústria de moda italiana. }\end{array}$ \\
\hline $\begin{array}{l}\text { Oliveira; Epaminondas } \\
\text { (2014) }\end{array}$ & $\begin{array}{l}\text { Descrever como o conhecimento, a inovação e a estratégia competitiva são } \\
\text { tratadas no contexto das organizações do setor atacadista da moda mineira. }\end{array}$ \\
\hline Kanat; Altigan (2014) & $\begin{array}{l}\text { Apresentar os efeitos da gestão do conhecimento sobre a gestão da cadeia } \\
\text { de abastecimento no setor do vestuário. }\end{array}$ \\
\hline Marcella; Rowley (2015) & $\begin{array}{l}\text { Investigar o grau de ferramentas e técnicas de gerenciamento de projeto } \\
\text { que podem ser aplicadas em toda a indústria efetivamente criativa, através } \\
\text { de um estudo de sua aplicação na indústria da moda no Nordeste da } \\
\text { Escócia. }\end{array}$ \\
\hline
\end{tabular}

Fonte: Elaborado pelos autores (2017)

Ao analisar os objetivos apresentados no Quadro 5, percebeu-se que Olaru et al. (2013) e Olaru, Ghituleasa e Filipescu (2012) são os únicos trabalhos que colocam como objetivo o de "examinar a gestão do conhecimento na indústria de moda", isso contribui para 
afirmar que há poucos estudos que tratam sobre a GC nessa perspectiva, e que a partir dos resultados encontrados por esses autores é possível sugerir direcionamentos a outros estudos, como específicos a determinado setor ou área no contexto dessas indústrias. Já os trabalhos de Li et al. (2005), Li, Xiaogang e Jun (2010), Kanat e Altigan (2014) trazem o termo "Gestão do Conhecimento" descrito nos objetivos, relacionado ao sentido de implantar esse sistema em empresas de moda. O que mostra mais uma vez a pertinência de discussão sobre a GC e o campo da moda/vestuário, por considerar que estes trabalhos não são suficientes para dar suporte a um entendimento amplo sobre $o$ assunto.

Os trabalhos publicados por Bertolini et al. (2004) e Kanat e Altigan (2014), tratam do conhecimento aplicado à cadeia de suprimentos na indústria do vestuário, segundo os autores, esse direcionamento se dá para entender como o conhecimento é utilizado de forma estratégica com foco em setores ligados à área produtiva. Isso apresenta uma nova perspectiva para o setor de moda/vestuário no campo das pesquisas, onde geralmente se percebe direcionamento maior para questões relacionadas à problematização (não menos importante) para soluções sobre os aspectos criativos ou no próprio desenvolvimento de produto, como se observa nos trabalhos de Marchi e Nardin (2009, 2014); Cavalcante et al. (2011); Oliveira e Epaminondas (2014) e Marcella e Rowley, (2015).

Weller (2007) apresenta a questão da complexidade existente no negócio de moda, para isto, propõe identificar as várias modalidades de conhecimento, que estão presentes no contexto da moda. Outro fator que é constantemente presente ao se tratar sobre produtos de moda/vestuário é a inovação. Neste sentido percebeu-se nas publicações de Marchi e Nardin (2009), Cavalcante et al. (2011) e Oliveira e Epaminondas (2014) que os autores trazem em seus objetivos discutir sobre o papel do conhecimento como um dos fatores ligados a inovação.

$\mathrm{Na}$ abordagem de Beirão Filho (2011), o autor buscou tratar a questão do compartilhamento de conhecimento, a partir do uso de ferramentas ligadas a tecnologia da informação e da comunicação. Discutindo como o uso dessas ferramentas podem proporcionar a criação de um sistema de informação que possibilite a captura e disseminação do conhecimento. De modo geral a ideia de explorar a GC no sentido de melhoria, inovação e uso da tecnologia é tratada por Wiig (1997), ao discorrer que um dos objetivos da GC é aprimorar e gerar competitividade. No mesmo viés sobre competitividade Jones (2005) trata que esse é um dos principais fatores que irá sustentar os negócios em empresas de moda e vestuário.

Para Olaru et al. (2013), o setor de moda e vestuário utiliza-se amplamente do uso do conhecimento individual, ou seja, do conhecimento tácito, isso está ligado ao tipo de negócio. Conforme verificou-se no referencial teórico o conhecimento tácito é o primeiro a estar disponível em um ambiente organizacional e nem sempre é fácil o tornar explícito (DALKIR, 2011; VASCONCELOS et al. 2012). Marchi e Nardin (2009; 2014) e Oliveira e Epaminondas (2014) discorrem que o conhecimento existente em empresas de moda e vestuário proporcionam a melhoria de seus processos contribuindo para a inovação em diferentes aspectos, isso vai ao encontro dos objetivos de uma sistemática de GC conforme observa-se nas contribuições de Dalkir (2001), CEN (2004) e Silva, Menegassi e Pegino (2017), principalmente no sentido de que a GC pode tornar organizações, pessoas e processos mais produtivos.

Quanto aos objetivos descritos nas publicações encontradas, portanto, sugere-se que há uma classificação seguindo três abordagens principais, que são: i) aplicação do conhecimento; ii) estratégia e conhecimento; e, iii) inovação e conhecimento. Nesse sentido sugere-se lacunas a serem respondidas em futuras pesquisas e publicações sobre o contexto da moda e vestuário, por exemplo, a fragmentação de processos descrita por Finardi, Silva e Varvakis (2016) e, como a GC pode contribuir na melhoria ou criação de valor para essas

Perspectivas em Gestão \& Conhecimento, João Pessoa, v. 8, n. 3, p. 192-208, set./dez. 2018. 
empresas. Ao verificar o interesse em estratégia e inovação isso vai ao encontro do que trata Jones (2005) e Silva, Menegassi e Pegino (2017) de que o setor de moda/vestuário se mantém competitivo por meio da criação e novidade de produtos.

Diante da proposta dos objetivos, foi necessário identificar qual o procedimento metodológico adotado pelos autores para alcançarem seus resultados. De fato, avaliar as metodologias utilizadas possibilita adotar possíveis trajetórias metodológicas que levem a estudos dentro da mesma temática. Deste modo, o Quadro 6 apresenta a metodologia utilizada nas publicações, distribuídas em: tipo de pesquisa, natureza, estratégia e técnica de coleta de dados.

Quadro 6 - Metodologia utilizada nos trabalhos

\begin{tabular}{|c|c|c|c|c|}
\hline Autor/Ano & Tipo de Pesquisa & $\begin{array}{c}\text { Natureza da } \\
\text { Pesquisa }\end{array}$ & Estratégia de Pesquisa & $\begin{array}{c}\text { Técnica de coleta de } \\
\text { dados }\end{array}$ \\
\hline Weller (2003) & Empírica & $\begin{array}{l}\text { Quantitativa/ } \\
\text { Qualitativa }\end{array}$ & Estudo de Caso & $\begin{array}{l}\text { Análise Documental e } \\
\text { Observação }\end{array}$ \\
\hline $\begin{array}{l}\text { Bertolini et al. } \\
(2004)\end{array}$ & Exploratória & Qualitativa & Pesquisa de Campo & $\begin{array}{l}\text { Entrevistas e reunião de } \\
\text { grupo }\end{array}$ \\
\hline $\begin{array}{l}\text { Eric et al. } \\
(2005) \\
\text { Eric et al. } \\
(2006)\end{array}$ & Empírica & Qualitativa & Pesquisa de Campo & Observação \\
\hline Weller (2007) & Teórica & Qualitativa & $\begin{array}{l}\text { Levantamento } \\
\text { bibliográfico }\end{array}$ & Análise bibliográfica \\
\hline $\begin{array}{l}\text { Balestrin; } \\
\text { Vargas e } \\
\text { Fayard, (2008) }\end{array}$ & Empírica & Qualitativa & Estudo de Caso & Observação e entrevista \\
\hline $\begin{array}{l}\text { Davey; } \\
\text { Schneider e } \\
\text { Davey (2009) }\end{array}$ & Teórica & Qualitativa & Revisão Sistemática & $\begin{array}{l}\text { Análise bibliográfica e } \\
\text { busca documental }\end{array}$ \\
\hline $\begin{array}{l}\text { Marchi; Nardin } \\
(2009)\end{array}$ & $\begin{array}{l}\text { Empírica/ } \\
\text { Exploratória }\end{array}$ & Qualitativa & Pesquisa de Campo & $\begin{array}{l}\text { Entrevista } \\
\text { semiestruturada }\end{array}$ \\
\hline $\begin{array}{l}\text { Fraganito } \\
(2010)\end{array}$ & Teórica & Qualitativa & $\begin{array}{l}\text { Levantamento } \\
\text { bibliográfico } \\
\end{array}$ & Análise bibliográfica \\
\hline Tres (2010) & Empírica & Qualitativa & Pesquisa de Campo & Entrevista \\
\hline $\begin{array}{l}\text { Li; Xiaogang e } \\
\text { Jun (2010) }\end{array}$ & Teórica & Qualitativa & $\begin{array}{l}\text { Levantamento } \\
\text { bibliográfico }\end{array}$ & Análise bibliográfica \\
\hline $\begin{array}{l}\text { Cavalcante et } \\
\text { al. (2011) }\end{array}$ & Exploratória & Qualitativa & Estudo de Caso & $\begin{array}{l}\text { Análise Documental e } \\
\text { entrevista } \\
\text { semiestruturada }\end{array}$ \\
\hline $\begin{array}{l}\text { Beirão Filho } \\
(2011) \\
\end{array}$ & Exploratória & Qualitativa & Estudo de Caso & Observação \\
\hline $\begin{array}{l}\text { Olaru; } \\
\text { Ghituleasa e } \\
\text { Filipescu (2012) }\end{array}$ & Exploratória & Qualitativa & $\begin{array}{l}\text { Levantamento } \\
\text { Bibliográfico e criação } \\
\text { de metodologia }\end{array}$ & $\begin{array}{l}\text { Análise bibliográfica e } \\
\text { aplicação de } \\
\text { ferramenta. }\end{array}$ \\
\hline Olszowy (2012) & Empírica & Qualitativa & Pesquisa de Campo & Observação \\
\hline Pires (2012) & Teórica & Qualitativa & $\begin{array}{l}\text { Levantamento } \\
\text { bibliográfico }\end{array}$ & Análise bibliográfica \\
\hline $\begin{array}{l}\text { Olaru et al. } \\
(2013)\end{array}$ & Exploratória & Qualitativa & $\begin{array}{l}\text { Levantamento } \\
\text { Bibliográfico e criação } \\
\text { de metodologia }\end{array}$ & $\begin{array}{l}\text { Análise bibliográfica e } \\
\text { aplicação de } \\
\text { ferramenta. }\end{array}$ \\
\hline
\end{tabular}

Perspectivas em Gestão \& Conhecimento, João Pessoa, v. 8, n. 3, p. 192-208, set./dez. 2018. 


\begin{tabular}{|l|l|l|l|l|}
\hline $\begin{array}{l}\text { Sohn; Vieira e } \\
\text { Filho, (2013) }\end{array}$ & Exploratória & Qualitativa & Pesquisa de Campo & $\begin{array}{l}\text { Observação e } \\
\text { questionário }\end{array}$ \\
\hline $\begin{array}{l}\text { Paulos; Moniz } \\
(2013)\end{array}$ & Exploratória & $\begin{array}{l}\text { Quantitativa/ } \\
\text { Qualitativa }\end{array}$ & Estudo de Caso & $\begin{array}{l}\text { Entrevista Exploratória e } \\
\text { semidiretiva }\end{array}$ \\
\hline $\begin{array}{l}\text { Marchi; Nardin } \\
(2014)\end{array}$ & Exploratória & Qualitativa & Pesquisa de Campo & Análise Documental \\
\hline $\begin{array}{l}\text { Oliveira; } \\
\text { (2014) }\end{array}$ & Exploratória & $\begin{array}{l}\text { Quantitativa/ } \\
\text { Qualitativa }\end{array}$ & Pesquisa de Campo & Entrevistas \\
\hline $\begin{array}{l}\text { Kanat; Altigan } \\
\text { (2014) }\end{array}$ & Exploratória & Quantitativa & Pesquisa de Campo & $\begin{array}{l}\text { Aplicação de } \\
\text { Questionário }\end{array}$ \\
\hline $\begin{array}{l}\text { Marcella; } \\
\text { Rowley (2015) }\end{array}$ & Exploratória & Qualitativa & Pesquisa de Campo & $\begin{array}{l}\text { Investigação e aplicação } \\
\text { de ferramentas }\end{array}$ \\
\hline
\end{tabular}

Fonte: Elaborado pelos autores (2017)

Ao analisar os procedimentos metodológicos utilizados pelos autores percebe-se que a pesquisa de campo se destaca enquanto estratégia de pesquisa. A pesquisa de campo prioriza a coleta de dados diretamente na fonte onde os fatos ocorrem, em geral para a coleta de dados utiliza-se principalmente da observação, aplicação de questionários e entrevistas, que visam comprovar uma hipótese ou responder a um problema (MARCONI; LAKATOS, 2003). Nessas publicações verificou-se que as principais técnicas de coleta de dados foram entrevistas, observação e aplicação de questionário.

Já o estudo de caso é a segunda estratégia de pesquisa mais utilizada nas publicações. Para Yin (2001) o estudo de caso tem por objetivo compreender fenômenos sociais complexos, proporcionando a preservação dos dados e fatos já que estes não poderão ser alterados pelo pesquisador. Para essas publicações as principais técnicas de coleta de dados são a observação, a entrevista e a análise documental. Os trabalhos de Weller (2003); Balestrin, Vargas e Fayard (2008), Cavalcante et al. (2011), Beirão Filho (2011) e Paulos e Moniz (2013) se utilizam dessa estratégia. Considerando o tratado por Jones (2005); Finardi, Silva e Varvakis (2016) e Silva, Menegassi e Pegino (2017), sobre a fragmentação de processos e envolvidos o estudo de caso possui tendência a solidificar as pesquisas de modo a melhor avaliar o fenômeno que se está estudando. No caso dessas indústrias o estudo de caso se mostra favorável por o pesquisador ter a possibilidade de limitar ou se aproximar melhor dos processos e ou dos envolvidos.

Por último nota-se que o levantamento bibliográfico foi a estratégia de pesquisa mais utilizada, esse tipo de pesquisa baseia-se na procura em materiais já publicados, para responder a um problema, mediante discussões já realizadas por outros autores (MARCONI; LAKATOS, 2003). A análise do Quadro 4 possibilitou ainda a verificação de que os estudos que se utilizam de dados estatísticos ou matemáticos são escassos, sendo que na maioria das publicações a natureza da pesquisa se caracteriza como qualitativa. E nesse sentido há a possibilidade de estudos que avaliem a GC por uma perspectiva de resultados, sendo que para isso seria necessário a utilização de modelos de GC ou uso de métricas, por exemplo (DALKIR, 2011). 


\section{CONSIDERAÇÕES FINAIS}

A análise nas publicações em periódicos nacionais e internacionais, bem como teses e dissertações encontradas nesta pesquisa, possibilitou compreender como é tratada a relação entre a GC e a área de moda e vestuário por pesquisadores em diferentes contextos.

As publicações se mostram em sua maioria escritas e publicadas em inglês, por pesquisadores de diferentes partes do mundo. A revisão sistemática de literatura possibilitou identificar que as publicações sobre o tema tiveram início no ano de 2003 percorrendo um caminho até o ano de 2015. Até o momento de conclusão da pesquisa - junho de 2016 - não houveram publicações sobre o tema. A primeira publicação em português foi do ano de 2009, enquanto que em inglês as publicações se iniciaram no ano de 2003, o que mostra retardo nas publicações sobre esse assunto escritas em português.

Ao avaliar as palavras-chave indicadas nas publicações os termos mais utilizados foram Knowledge Management e Gestão do Conhecimento e, quanto à a associação ao tipo de empresa, têm-se Fashion, Clothing, Moda e Vestuário, termos esses que foram utilizados como palavras-chave para a busca das publicações apresentadas neste trabalho e mostram os principais conceitos referentes ao assunto.

Os objetivos das pesquisas se mostram variados, no entanto percebeu-se que poucas publicações trazem como objetivo aplicar a GC a setores específicos de empresas de moda e vestuário; há certa tendência para objetivos que tratem do processo criativo ou de desenvolvimento de produto. Quanto aos objetivos foi possível criar três grupos que contêm as principais abordagens, sendo: aplicação do conhecimento; estratégia e conhecimento; e, inovação e conhecimento.

Quanto à metodologia, foi possível observar que a pesquisa de campo corresponde a grande parte das estratégias de pesquisa, seguida pelos estudos de caso e levantamento bibliográfico. Quanto às técnicas de coleta de dados, a análise bibliográfica e a análise documental são as mais utilizadas pelos autores, seguidas de entrevistas e questionários.

Este trabalho considera a GC uma ferramenta estratégica que pode ser utilizada pelas organizações como meio para criação de valor, inovação de processos e produtos e tornar as pessoas mais produtivas. No entanto as pesquisas que tratam sobre essa temática e sua relação com as indústrias ligadas à moda e ao vestuário são poucas. Percebeu-se que mesmo que alguns autores possuam mais de uma publicação sobre a mesma temática, não há continuidade das pesquisas, ou seja, o setor de moda/vestuário se torna carente de pesquisas que visem potencializar sua importância estratégica na economia brasileira. Mesmo considerando todos os setores envolvidos nos processos de empresas de moda/vestuário, as publicações apresentam poucas pesquisas que tratam sobre setores ligados à área produtiva, como o setor de compras, almoxarifado, o de costura e o de expedição, por exemplo.

Por fim, esta pesquisa mostra que há necessidade de discussões em torno da GC e sua relação com o setor de moda/vestuário. Que há lacunas ainda a serem discutidas em demais pesquisas da mesma modalidade ou não, que devido as características específicas do setor de moda e vestuário os estudos de caso podem apresentar resultados importantes por possibilitar uma melhor aproximação com a estrutura dessas empresas.

\section{REFERÊNCIAS}

ABIT. Associação Brasileira das Indústrias Têxteis e de Vestuário. Perfil do Setor: dados gerais do setor atualizados em 2016, referentes ao ano de 2015. Disponível em http://www.abit.org.br/cont/perfil-do-setor. Acesso em: 15 de julho de 2016.

Perspectivas em Gestão \& Conhecimento, João Pessoa, v. 8, n. 3, p. 192-208, set./dez. 2018. 
BALESTRIN, A.; VARGAS, L. M.; FAYARD, P. Knowledge creation in small-firm network. Journal of Knowledge Management, v. 12, n. 2, p. 94-106, 2008.

BAXTER, M. Projeto de produto: guia prático para o design de novos produtos. São Paulo: Edgard Blücher, 1998.

BEIRÃO FILHO, J. A. Criação e compartilhamento do conhecimento da área de moda em um sistema virtual integrado de informações. 166 p. Tese (doutorado) - Programa de PósGraduação em Engenharia e Gestão do Conhecimento. Universidade Federal de Santa Catarina, Centro Tecnológico. Florianópolis, 2011.

BERTOLINI, M.; BEVILACQUA, M.; BOTTANI, E.; RIZZI, A. Requirements of an ERP enterprise modeller for optimally managing the fashion industry supply chain. Journal of Enterprise Information Management, v. 17, n. 3, p. 180-190, 2004.

CAVALCANTE, L. de Q. B.; MURAKAMI, L.C.; REINALDO, H. O. A.; PINTO, S. dos S. D.; SILVA FILHO, J. C. L. da. A abordagem prospectiva de gestão do conhecimento na indústria da moda: um modelo para o aumento de inovação de produto. Biblioteca Digital de la Asociación Latino-Iberoamericana de Gestión Tecnológica, v. 1, n. 1, [s.p], 2013.

CEN. European Guide to Good Practice in Knowledge Management. Management Science, Brussels, v. 50, n. 3, p. 352-364, 2004.

DALKIR, K. Knowledge Management in Theory and Practice. London: The MIT Press, 2011.

DAVEY, J.; SCHNEIDER, L.; DAVEY, H. Intellectual capital disclosure and the fashion industry. Journal of Intellectual Capital, v. 10, n. 3, p. 401-424, 2009.

FINARDI, C.; SILVA, E. L. D.; RADOS, G. J. V. O fluxo da informação no processo de design de moda: uma análise aplicada em pequenas empresas de confecção da grande Florianópolis Santa Catarina. Perspectivas em Gestão \& Conhecimento, João Pessoa, v. 6, n. 2, p. 134-152, jul/dez 2016.

FRAGANITO, R. M. F. Cooperação e Inovação no Processo de Criação e Gestão do Conhecimento no sector Têxtil e de Vestuário. Instituto Politécnico de Lisboa; Repositório Científico, p.1-56, 2010.

GIL, A. C. Como elaborar projetos de pesquisa. 5. ed. São Paulo. Atlas, 2010.

JONES, S. J. Fashion Design - Manual do Estilista. São Paulo: Cosac Naify, 2005.

KANAT, S.; ATILGAN, T. Effects of Knowledge Management on Supply Chain Management in the Clothing Sector: Turkish Case. Fibres \& Textiles in Eastern Europe, v. 22, n. 1, p. 9-13, 2014.

LASTRES, H. M. M.; FERRAZ, J. C. Economia da Informação, do Conhecimento e do Aprendizado. In: LASTRES, H. M. M.; ALBAGLI, S. Informação e globalização na era do conhecimento. Rio de Janeiro: Campus, 1999. 
LI, E. P. H; TO, C. K. M; WONG, Y. H; CHANG, J. M. T. Managing Knowledge in Fashion Businesses: A Contextual Review. Research Journal of Textile and Apparel, v. 9, n. 2, p. 97-104, 2005.

LI, E. P. H; TO, C. K. M; WONG, Y. H; CHANG, J. M. T. Managing Knowledge in Fashion Businesses: A Survey of Contemporary Tools for Coordination and Collaboration. Research Journal of Textile and Apparel, v. 10, n. 2, p. 83-92, 2006.

LI, L.; XIAOGANG, L.; JUN, L. Apparel Brand Image Management Based on Knowledge Management. In: Management and Service Science (MASS), International Conference on. IEEE, p. 1-4 2010.

MARCELLA, M.; ROWLEY, S. An exploration of the extent to which project management tools and techniques can be applied across creative industries through a study of their application in the fashion industry in the North East of Scotland. International Journal of Project Management, v. 33, n. 4, p. 735-746, 2015.

MARCHI, G.; NARDIN G. Market Knowledge Transfer and Time Pressure in new Product Development: The Emergent Role of Knowledge Intermediaries in Fashion Industry. European Conference on Knowledge Management, 10 ${ }^{\text {th }}$., ECKM 2009, Vicenza, v. 2, p. 520-528, 2009.

MARCHI, G.; NARDIN G. Organizing Creativity in the Italian Fashion Industry. International Studies of Mgt. \& Org., v. 44, n. 1, p. 7-20, 2014.

MARCONI, M. de A.; LAKATOS, E. M. Fundamentos da Metodologia Científica. 5. ed. São Paulo. Atlas, 2003.

MARCONI, M. de A.; LAKATOS, E. M. Metodologia do Trabalho Científico. 6. ed. Reimp. São Paulo. Atlas, 2006.

OLARU, S; GHITULEASA, P. C.; FILIPESCU E. Knowledge Management in the Fashion Industry. EUROPEAN CONFERENCE ON KNOWLEDGE MANAGEMENT, $13^{\text {th }}$., Universidad Politécnica de Cartagena, Spain, v. 1, p. 6-7, Sep. 2012.

OLARU, S.; FILIPESCU, E.; SPINACHE, E.; GEORGETA, P. Collaborative Technologies and Knowledge Management in the Fashion Industry. Annals... of The University Of Oradea Fascicle Of Textiles, Leatherwork. v. XIV, n. 1, p. 26-32, 2013.

OLIVEIRA, P. H. de; EPAMINONDAS, M. E. R. Conhecimento, Inovação e Estratégia competitiva: um estudo no setor atacadista da moda. Revista Eletrônica de Estratégia \& Negócios, v. 7, n. 1, p. 82-104, 2014.

OLSZOWY, M. M. Importance of Intellectual Capital in Enterprise Growth, with Special Emphasis on the Textile and Clothing Industry in Poland. Fibres \& Textiles in Eastern Europe, n. 5, p. 10-15, 2012.

PAULOS, M. R.; MONIZ, A. B. Os trabalhadores do conhecimento num setor tradicional: o caso dos designers do vestuário. Sociologia, Problemas e Práticas, n. 72, p. 103-122, 2013. 
PIRES, V. S. Cultura organizacional e memória empresarial na indústria da moda: gestão de ativos do conhecimento como estratégia corporativa para a construção de identidades de marcas. e-Com, v. 5, n. 2, [s.p], 2012.

SILVA, M.J; MENEGASSI, C.H.M.; PEGINO, P.M.F. Análise de estudos sobre a gestão do conhecimento como fator de estratégia nas indústrias de moda/vestuário. Revista Conbrad, v. 2, n. 3, p. 35-48, 2017.

SOHN, A. P. L.; VIEIRA, F. D.; CASAROTTO FILHO, N. Estudo comparativo de canais de transmissão de conhecimento em clusters têxteis e de vestuário de Santa Catarina-Brasil e do Norte de Portugal. Repositório Uminho, 2013. Disponível em: http://hdl.handle.net/1822/36764. Acesso em: 15 de maio de 2016.

TRES, J. A dinâmica dos fluxos de conhecimentos locais e externos no sistema local de produção têxil-vestuário catarinense. 204 p. Tese (Doutorado) Programa de Doutorado em Engenharia de Produção - Escola Politécnica, Universidade de São Paulo, São Paulo, 2010.

VASCONCELOS, K. C. de A.; MERHI, D. Q.; SILVA JUNIOR, A da; SILVA, P. de O. M. da. Cultura, Liderança e Compartilhamento do Conhecimento Organizacional. Revista de Administração FACES Journal, v. 12, n. 1, 2013.

WIIG, K. M. Knowledge Management: Where Did It Come From and Where Will It Go? Expert Systems With Application. v. 13, n. I, p. 1-14, 1997.

WELLER, S. A. Fashion's influence on garment mass production: Knowledge, commodities and the capture of value. $347 \mathrm{f}$. Tese (Doutorado). Centro de Estudos Económicos Estratégicos Victoria University, Melbourne, Austrália, 2003.

WELLER, S. Fashion as viscous knowledge: fashion's role in shaping trans-national garment production. Journal of Economic Geography, v. 7, n. 1, p. 39-66, 2007.

YIN, R. K. Estudo de caso: planejamento e métodos. Tradução de Daniel Grassi. 2. ed. Porto Alegre: Bookman, 2001.

Artigo recebido em 31/03/2017 e aceito para publicação em 07/10/2018 